\title{
ACR Proton Acceleration Associated with Reconnection Processes beyond the Heliospheric Termination Shock
}

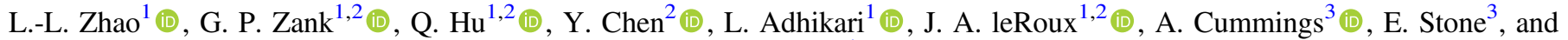 \\ L. F. Burlaga ${ }^{4}$ (1) \\ ${ }^{1}$ Center for Space Plasma and Aeronomic Research (CSPAR), The University of Alabama in Huntsville, Huntsville, AL 35805, USA; lz0009@uah.edu, \\ gary.zank@uah.edu \\ ${ }^{2}$ Department of Space Science, The University of Alabama in Huntsville, Huntsville, AL 35899, USA \\ ${ }^{3}$ California Institute of Technology, Mail Code 290-17, Pasadena, CA 91125, USA \\ ${ }^{4}$ NASA Goddard Space Flight Center, Code 673, Greenbelt, MD 20771, USA \\ Received 2019 August 26; revised 2019 October 10; accepted 2019 October 13; published 2019 December 2
}

\begin{abstract}
One of the curious observations from the Voyagers is that the intensity of anomalous cosmic rays (ACRs) did not peak at the heliospheric termination shock (HTS) but instead a short distance (within $\sim 1$ au) downstream of the HTS. One possible explanation is that the interaction of the wavy heliospheric current sheet with the HTS enhances magnetic reconnection and generates numerous small-scale magnetic flux ropes in the heliosheath immediately downstream of the HTS. Charged particles are accelerated in this region due to Fermi acceleration and the reconnection electric field. In this work, we provide observational evidence of the presence of magnetic flux ropes in the heliosheath region just downstream of the HTS using a wavelet analysis of the reduced magnetic helicity and Grad-Shafranov reconstruction techniques. The Zank et al. kinetic transport theory for particles propagating through the magnetic islands region is employed to fit the observed energetic proton intensities in the post-HTS region. Our modeling results agree reasonably well with the observations, which suggests that stochastic acceleration via reconnection processes can explain the ACR proton peak beyond the HTS.
\end{abstract}

Unified Astronomy Thesaurus concepts: Interplanetary particle acceleration (826); Solar magnetic reconnection (1504); Termination shock (1690); Solar wind (1534)

\section{Introduction}

The heliospheric termination shock (HTS) is usually considered as the source location of anomalous cosmic rays (ACRs), where interstellar pickup ions (PUIs) were thought to experience diffusive shock acceleration (DSA; e.g., Pesses et al. 1981). This conventional model of ACR generation was widely accepted until the Voyager spacecraft crossings of the HTS on 2004 December 16 and 2007 August 30, respectively (Stone et al. 2005, 2008). The ACR intensity profiles from both Voyager 1 (V1) and Voyager 2 (V2) measurements did not peak at the HTS (Decker et al. 2005; Cummings \& Stone 2008, 2013; Senanayake et al. 2015) as predicted. Instead, the ACR intensity peaked behind the HTS, within about $1 \mathrm{au}$. In this paper, we address the origin of peak behind the HTS and argue that it is due to an acceleration process different from classical DSA. We remark that on much larger scales $(>10 \mathrm{au})$ the ACR intensity continued to increase beyond the HTS and peaked deep in the inner heliosheath. This is not the focus of this paper. The origin of ACRs and the role of the HTS is an outstanding problem of heliospheric physics. Similar behavior has been observed downstream of quasi-perpendicular interplanetary shocks in the vicinity of the heliospheric current sheet (HCS; e.g., Zhao et al. 2018, 2019b). Although models have been proposed to study the large-scale enhancement of the ACR intensity over distances greater than $\sim 10$ au (e.g., McComas \& Schwadron 2006; Zhang 2006; Ferreira et al. 2007), there has been very little discussion about the observed ACR distribution during the HTS crossing by Voyager 2 and how it relates to classical DSA. Drake et al. (2010) proposed that ACRs might be accelerated by some form of magnetic reconnection in the sectored field region within the heliosheath. The folded HCS creates the sectored magnetic field (Burlaga et al. 2005, 2006), which piles up on its approach to the heliopause. As the current sheets are compressed, Drake et al. suggest that magnetic reconnection is more likely to occur in this region than in the supersonic solar wind. However, as illustrated during the Voyager 1 heliopause crossing, there was no evidence of a pileup or peaking of ACRs before the crossing, and in fact the energetic ACRs exhibited a flat profile as the heliopause was approached. Nonetheless, that the observed energetic ions in the heliosheath can be produced by capturing the magnetic energy released from magnetic reconnection has some observational support (e.g., Hill et al. 2014). The simulations of reconnection by Drake et al. in the sectored field within the heliosheath suggested that most energetic ions gain energy through Fermi reflection in interacting islands. Guided by these numerical simulation results, comprehensive kinetic transport theories were developed to model reconnection associated particle acceleration in solar wind regions filled with numerous interacting magnetic islands (Zank et al. 2014; le Roux et al. 2015 , 2018). In these theories, first-order and second-order Fermi processes, and "antireconnection" electric field acceleration are thought to play an important role in the energization of charged particles.

From analysis of $A C E$, and Wind at $1 \mathrm{au}$, and Ulysses data at $5 \mathrm{au}$, a number of energetic particle events, which are inconsistent with a 1D stationary DSA theoretical model, have been ascribed to reconnection-based particle acceleration in a dynamic multi-islands environment (Khabarova \& Zank 2017; Zhao et al. 2018, 2019b; Adhikari et al. 2019). Using the analytical solution derived from the Zank et al. stochastic transport theory, Zhao et al. (2018) and Adhikari et al. (2019) obtained good theoretical fits to the observed unusual energetic ion events at 1 and 5 au by assigning a dominant role to first- 
order Fermi acceleration during the contraction and reconnection of islands. Their model results reproduced several typical characteristics for the particle distribution. These include the particle flux peaking behind the shock instead of the shock front, a flux amplification factor that increases with particle energy, and particle spectra that harden with increasing distance through the island's acceleration region. By combining both DSA and magnetic-island-reconnection-related processes, Zank et al. (2015) explained the Voyager 2 ACR proton flux enhancement beyond the HTS.

Although reconnection acceleration due to magnetic islands dynamics is one possible explanation for the observed ACR proton flux peak behind the HTS, the presence of magnetic islands in the heliosheath remains to be confirmed. This is a key question that we investigate in this paper. We show the presence of magnetic islands downstream of the HTS and simultaneous particle acceleration in this region.

Early observations identified magnetic island or flux rope structures by rotation of the magnetic field (Moldwin et al. 1995). Recently, more advanced techniques have been developed to identify small-scale magnetic flux ropes in the solar wind. Zheng \& Hu (2018) and Hu et al. (2018) developed an automatic scheme that detects small-scale magnetic flux ropes based on a Grad-Shafranov (GS) reconstruction. Another method is to use magnetic helicity as a proxy for a magnetic flux rope. The strict definition of magnetic helicity should be the volume integral of the dot product between the magnetic field strength and the vector potential (Matthaeus et al. 1982; Telloni et al. 2012, 2013). This of course depends on the spatial properties of the magnetic field topology, which cannot be evaluated from single spacecraft measurements. Matthaeus et al. (1982) described a reduced form of magnetic helicity that can be estimated in the frequency domain based on the magnetic power spectrum. Since magnetic flux ropes possess helical magnetic field lines, they typically have a large magnetic helicity. Using wavelet analysis, Telloni et al. (2013) studied the time evolution of the reduced magnetic helicity spectrum, and found that magnetic flux ropes at 1 au exhibit a good correspondence with high magnetic helicity. Cross-helicity and residual energy spectra can also be used to distinguish flux ropes from Alfvénic structures (Adhikari et al. 2017; Zank et al. 2017).

In this paper, we first present an overview of the Voyager 2 magnetic field and plasma observations of the HTS crossing. Second, we show the magnetic helicity, cross-helicity, and residual energy spectra based on a Morlet wavelet analysis to identify magnetic island/flux rope structures in the inner heliosheath. Finally, the observed ACR proton "time-intensity" profiles immediately downstream of the HTS are quantitatively compared with our reconnection acceleration model.

\section{Overview of Voyager 2 HTS Crossing}

Figure 1 displays the time evolution of the energetic proton flux, magnetic field, and plasma properties as measured by Voyager 2 during the period between 2007 May 1 and 2008 May 1. The panels from top to bottom show, respectively, the daily averaged $10.3-12.8 \mathrm{MeV}$ ACR proton flux with the measurement uncertainty included, the heliospheric magnetic field (HMF) strength $|B|$, the elevation $(\theta)$ and azimuthal $(\phi)$ angles of the magnetic field direction in the RTN coordinate system, the bulk flow speed $V_{\mathrm{sw}}$, proton density $n_{\mathrm{p}}$ and temperature $T_{\mathrm{p}}$, thermal, magnetic, and total pressure, and the ratio of thermal pressure to magnetic pressure $(\beta)$. All the parameters have been averaged over 1 day. The proton flux and plasma beta are plotted on a log scale. During this period, Voyager 2 crossed the HTS at $\sim 84$ au on 2007 August 30 (Burlaga et al. 2008; Stone et al. 2008), as indicated by the dashed vertical line in each panel. The HTS crossing was characterized by an abrupt drop in bulk flow speed from supersonic to subsonic. The downstream flow speed is around $\sim 150 \mathrm{~km} \mathrm{~s}^{-1}$, which is subsonic due to the modification of the sound speed caused by PUIs (Florinski et al. 2009; Mostafavi et al. 2017; Zank et al. 2018). The crossing is also accompanied by increases in proton density, temperature, and pressure. The daily averaged HMF strength did not show a large increase for $\sim 60$ days after the HTS crossing. Burlaga et al. (2008) show the increase in magnetic field strength during three HTS crossing with $48 \mathrm{~s}$ averaged magnetic field data, and they suggest that the HTS is a complex, quasi-perpendicular shock with moderate strength. Burlaga et al. (2009a) identified two "merged interaction regions" (MIRs) around 2007 June 4 (day 155) and 2007 July 20 (day 201) before the HTS crossing, which are characterized by enhanced magnetic field strength $|B|$, magnetic pressure, and low values of the plasma beta. We do not address the corresponding changes in energetic proton flux associated with these large-scale structures before the HTS crossing, but focus exclusively on the sustained enhancement of proton flux downstream of the HTS. After the crossing of the HTS, the proton density, temperature, total pressure, and plasma beta all increase significantly and exhibit a high level of fluctuations in the heliosheath. The proton density and temperature are highly correlated. The magnetic field fluctuates rapidly with a small amplitude in the immediate downstream region and has a large amplitude further downstream. The magnetic field direction changes frequently behind the HTS as shown in the HMF elevation $\theta$ and azimuthal $\phi$ panels. At around 2008 January 1, a unidirectional magnetic field appears and lasts for about 80 days, which is identified as the unipolar region and may be due to the movement of the HCS to the equatorial plane (Burlaga et al. 2009a). The plasma beta in the downstream heliosheath region reaches 10 or more, and changes dramatically. Note that in calculating the plasma beta, only the thermal plasma is included and the hotter pickup ions (Zhao et al. 2019a), which cannot be measured, are neglected. Previous studies suggest that the thermal plasma remains relatively cold on transmission through the HTS and it is the PUIs that carry much of the energy (Zank et al. 1996, 2010, 2018; Mostafavi et al. 2017, 2018). Therefore, the value of the plasma beta calculated in the downstream heliosheath region is probably very much a lower bound due to the neglect of pickup ions. The 10.3-12.8 MeV ACR proton flux does not show a clear increase ahead of the HTS. Instead, the downstream flux exhibits a long-lasting steady increase until early December 2007 followed by a decrease until 2008 January 1 . This is different from the predictions of conventional 1D stationary DSA, which predicts that the particle intensity downstream of the shock should be constant with distance. The plasma beta, proton density, and temperature often show a local increase in the post-HTS region, indicating the possible existence of current sheets or reconnection exhausts that bound small-scale flux ropes/islands (Zheng \& $\mathrm{Hu}$ 2018). It has been suggested that the HTS may generate high levels of MHD turbulence, waves, vortices, and coherent structures downstream (Zank et al. 2006b; Burlaga \& Ness 2009; 


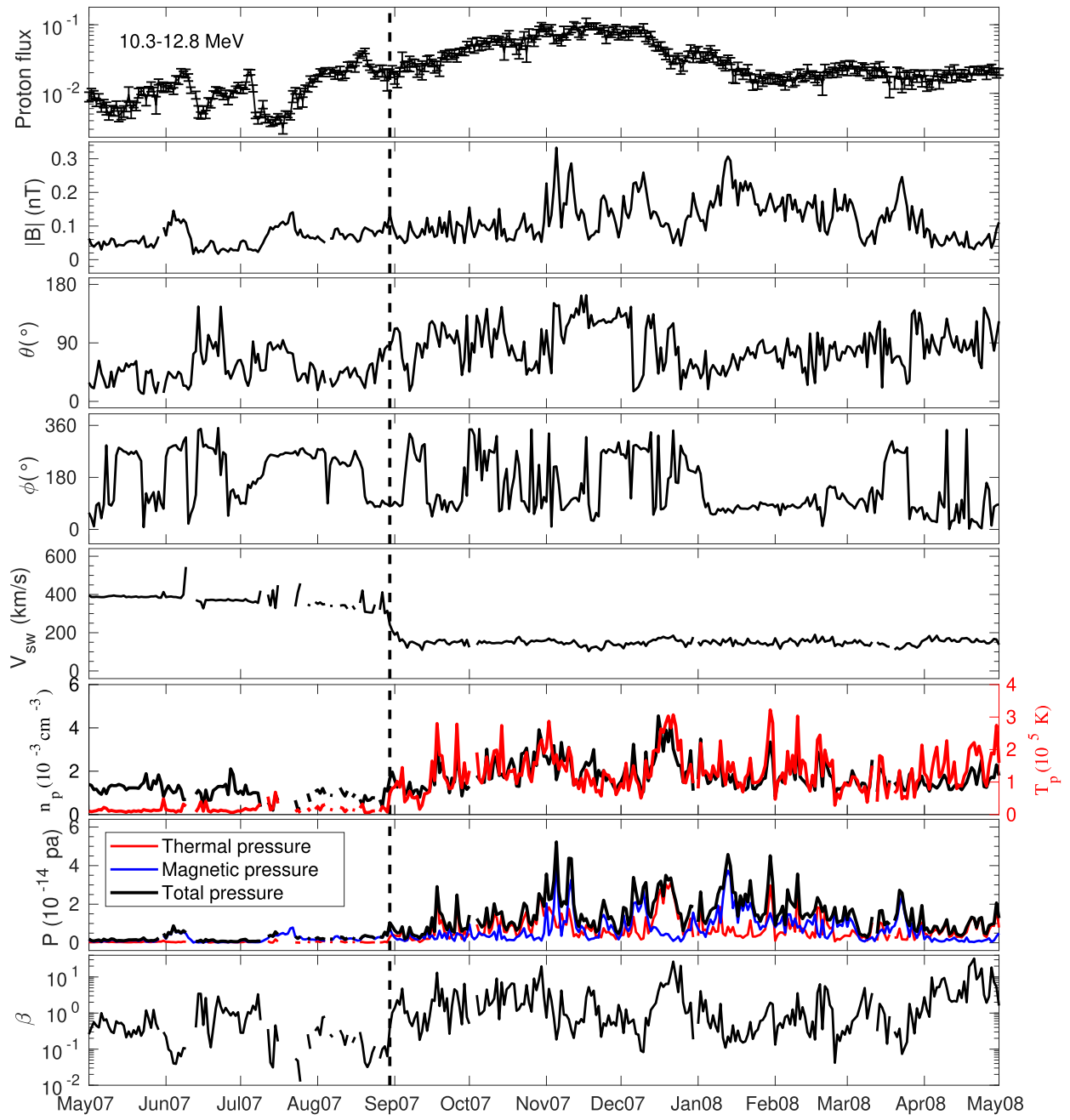

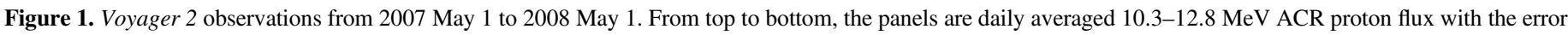

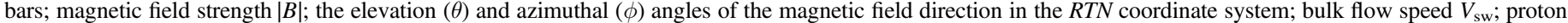

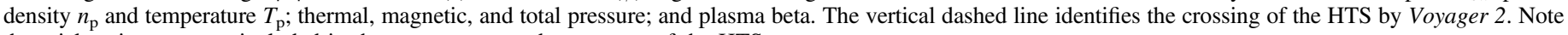
that pickup ions are not included in the measurements downstream of the HTS.

Burlaga et al. 2009b; Zank et al. 2015, 2018). Furthermore, the HTS at least in the region crossed by Voyager 2 may be located in the vicinity of interplanetary sector boundaries, i.e., the wavy $\mathrm{HCS}$, due to the observed rapid changes in magnetic field direction. The interaction of the HTS and the HCS may trap flux ropes/islands that are formed downstream of the HTS and produce more structures because of an increased reconnection rate and strong compression (Khabarova et al. 2015). All these factors suggest that island-related reconnection-based particle acceleration downstream of the HTS is likely to be important.

\section{Identification of Magnetic Islands}

Magnetic flux ropes/islands are defined by a magnetic field configuration corresponding to helical field lines winding around a central axis, and are thus expected to possess a high value of magnetic helicity. In order to investigate the possible existence of magnetic islands/flux ropes in the post-HTS region, we follow the method of Telloni et al. (2012) and use a Morlet wavelet analysis to study the signatures of three turbulence quantities in the inner heliosheath, namely magnetic helicity, cross-helicity, and residual energy. To evaluate these three turbulence quantities, we use the Elsässer variables, defined as:

$$
\boldsymbol{z}^{ \pm}=\boldsymbol{u} \pm \boldsymbol{b} / \sqrt{4 \pi n_{\mathrm{p}} m_{\mathrm{p}}}
$$

where $\boldsymbol{u}$ represents the fluctuating velocity field, $\boldsymbol{b}$ represents the fluctuating magnetic field, $n_{\mathrm{p}}$ is the proton density, and $m_{\mathrm{p}}$ is proton mass. Fluctuating magnetic and velocity fields can be separated from their mean fields as $\underline{\boldsymbol{B}}=\boldsymbol{B}_{0}+\boldsymbol{b} ; \underline{\boldsymbol{U}}=\boldsymbol{U}_{0}+\boldsymbol{u}$. Here, $\boldsymbol{B}_{0}$ is the mean magnetic field, $\boldsymbol{U}_{0}$ is the mean velocity field, $\langle\underline{\boldsymbol{B}}\rangle=\boldsymbol{B}_{0}$ and $\langle\boldsymbol{b}\rangle=0$, and so too with the velocity field. The Elsässer variables $z^{+}\left(z^{-}\right)$represent the forward (backward) propagating modes with respect to the HMF orientation. We then perform the Wavelet transforms (Torrence \& Compo 1998) on each component of the fluctuating magnetic field $b_{R}, b_{T}, b_{N}$. The normalized reduced magnetic helicity can be estimated by Matthaeus et al. (1982), Telloni et al. (2013), and Vasquez et al. (2018)

$$
\sigma_{\mathrm{m}}(\nu, t)=\frac{2 \operatorname{Im}\left[W_{T}^{*}(\nu, t) \cdot W_{N}(\nu, t)\right]}{\left|W_{R}(\nu, t)\right|^{2}+\left|W_{T}(\nu, t)\right|^{2}+\left|W_{N}(\nu, t)\right|^{2}},
$$


where $\nu$ is the frequency associated with the Wavelet function and the sampling period of the measured magnetic field in the $R T N$ coordinate system. The spectra $W_{R}(\nu, t), W_{T}(\nu, t)$, and $W_{N}(\nu, t)$ are the wavelet transforms of time series of $b_{R}, b_{T}$, and $b_{N}$, respectively, and $W_{T}^{*}(\nu, t)$ is the conjugate of $W_{T}(\nu, t)$. From the spectrogram of the magnetic helicity $\sigma_{\mathrm{m}}$, one can determine both the magnitude and the handedness of underlying fluctuations at a specific scale. A positive value of $\sigma_{\mathrm{m}}$ corresponds to right-handed chirality and a negative value to left-handed chirality.

The normalized cross-helicity $\sigma_{\mathrm{c}}$ and residual energy $\sigma_{\mathrm{r}}$ are calculated from the Elsässer variables $z^{ \pm}$(e.g., Zank et al. 2012):

$$
\sigma_{\mathrm{c}}=\frac{\left\langle z^{+2}\right\rangle-\left\langle z^{-2}\right\rangle}{\left\langle z^{+2}\right\rangle+\left\langle z^{-2}\right\rangle}
$$

and

$$
\sigma_{\mathrm{r}}=\frac{2\left\langle z^{+} \cdot z^{-}\right\rangle}{\left\langle z^{+2}\right\rangle+\left\langle z^{-2}\right\rangle}
$$

where $\left\langle z^{+2}\right\rangle$ and $\left\langle z^{-2}\right\rangle$, respectively, represent the energy density in forward and backward propagating modes. The absolute value of $\sigma_{\mathrm{m}}, \sigma_{\mathrm{c}}$, and $\sigma_{\mathrm{r}}$ are no more than $1 . \sigma_{\mathrm{c}}$ indicates the alignment between $\boldsymbol{b}$ and $\boldsymbol{u}$. A single Alfvén wave mode usually has a high value of $\left|\sigma_{\mathrm{c}}\right|$ (close to 1$)$. $\sigma_{\mathrm{r}}$ represents the energy difference between the fluctuating kinetic and magnetic energies. Magnetic fluctuating energy dominates when $\sigma_{\mathrm{r}}<0$, and kinetic fluctuating energy dominates when $\sigma_{\mathrm{r}}>0$. We further perform the wavelet transform $\mathcal{W}$ on the three components of the Elsässer variables $z_{R}^{ \pm}, z_{T}^{ \pm}$, and $z_{N}^{ \pm}$. The normalized residual energy $\sigma_{\mathrm{r}}$ and cross-helicity $\sigma_{\mathrm{c}}$ can be rewritten in both the frequency and time domains as in the wavelet transform. Scales greater than the COI are subject to edge effects, making these results not truly reliable. However, edge effects can be eliminated by increasing the window length of the analysis. The contour lines in the $\sigma_{\mathrm{m}}$ panel bound high magnetic helicity regions with $\left|\sigma_{\mathrm{m}}\right|>0.6$, which we considered to be four possible flux ropes downstream of the HTS. The position of the peak of $\left|\sigma_{\mathrm{m}}\right|$ in the scale domain indicates the duration of one particular flux rope, and the peak in the time domain suggests its central time. Table 1 lists the $\sigma_{\mathrm{m}}, \sigma_{\mathrm{c}}$, and $\sigma_{\mathrm{r}}$ values, as well as the central time and scale of these four structures. According to the value of $\sigma_{\mathrm{m}}$, structures A, B, and D have a left-hand chirality, and structure $\mathrm{C}$ has a right-hand chirality. The absolute value of the normalized cross-helicity $\left|\sigma_{\mathrm{c}}\right|$ is generally smaller than 0.3 in these four structures, which further suggests that they are likely to be magnetic flux ropes rather than Alfvénic structures. The normalized residual energy $\sigma_{\mathrm{r}}$ is negative for all four structures, especially for structures B and D, and they both have a high negative value of $\sigma_{\mathrm{r}}$. The negative $\sigma_{\mathrm{r}}$ indicates that magnetic fluctuation energy dominates, thus pointing to magnetic flux rope structures rather than fluid vortices.

The bottom two panels of Figure 2 show a GS reconstructed cross-section map and $P_{t}$ versus $A$ curve for a flux rope identified in the downstream heliosheath region. Here, $P_{t}$ is the total transverse pressure defined by $P_{t}=p+B_{z}^{2} / 2 \mu_{0}$, the sum of the plasma pressure and axial magnetic pressure, and $A(x, y)$ is the magnetic flux function. The detailed description of the GS reconstruction technique has been explained in several previous studies (e.g., Hu et al. 2018; Zheng \& Hu 2018; Zhao et al. $2019 b)$. In the left bottom panel, the cross-section is plotted with the transverse magnetic field (black contour lines) and the axial magnetic field $B_{z}$ (shaded color). The flux rope center corresponding to the maximum of $B_{z}$ is denoted by the white dot, and the projected field vectors along the spacecraft path are shown by white arrows. The right bottom panel shows the calculated $P_{t}$

$$
\sigma_{\mathrm{r}}(\nu, t)=\frac{2 \operatorname{Re}\left[\mathcal{W}^{*}\left(z_{R}^{+}\right) \cdot \mathcal{W}\left(z_{R}^{-}\right)+\mathcal{W}^{*}\left(z_{T}^{+}\right) \cdot \mathcal{W}\left(z_{T}^{-}\right)+\mathcal{W}^{*}\left(z_{N}^{+}\right) \cdot \mathcal{W}\left(z_{N}^{-}\right)\right]}{W^{+}(\nu, t)+W^{-}(\nu, t)}
$$

and

$$
\sigma_{\mathrm{c}}(\nu, t)=\frac{W^{+}(\nu, t)-W^{-}(\nu, t)}{W^{+}(\nu, t)+W^{-}(\nu, t)}
$$

where $W^{+}(\nu, t)$ and $W^{-}(\nu, t)$ represent the wavelet power spectrum in $z^{+}$and $z^{-}$modes, respectively. Thus, $W^{+}(\nu, t)=$ $\left|\mathcal{W}\left(z_{R}^{+}\right)\right|^{2}+\left|\mathcal{W}\left(z_{T}^{+}\right)\right|^{2}+\left|\mathcal{W}\left(z_{N}^{+}\right)\right|^{2}$ and $W^{-}(\nu, t)=\left|\mathcal{W}\left(z_{R}^{-}\right)\right|^{2}+$ $\left|\mathcal{W}\left(z_{T}^{-}\right)\right|^{2}+\left|\mathcal{W}\left(z_{N}^{-}\right)\right|^{2}$.

In the top panel of Figure 2, we analyzed the normalized magnetic helicity $\sigma_{\mathrm{m}}$, cross-helicity $\sigma_{\mathrm{c}}$, and residual energy $\sigma_{\mathrm{r}}$ to identify possible downstream structures via daily averaged magnetic field and plasma fluctuations observed by Voyager 2 during the period between 2007 August 30 (day 242 of 2007) and 2007 December 31 (day 365 of 2007). The location and scales of these possible flux ropes/islands are characterized by using wavelet spectrograms of these turbulence quantities. The wavelet scales are chosen to be 2-32 days in this study, which roughly represents the scale size of the structures. Due to limited time resolution of the observations ( $24 \mathrm{hr}$ resolution), it is not possible to study structures on smaller scales. The shaded cross area in the bottom of each panel is determined by the cone of influence (COI) versus $A$ as blue circles and orange dots for the two branches (coming into and out of the flux rope, respectively). $P_{t}$ versus $A$ fitting curve is shown by the black solid curve. The fitting residue $R_{f}=0.08$, which indicates the quality of double-folding pattern (Hu et al. 2018; Chen et al. 2019). The vertical line denoted by $A_{b}$ indicates the boundary of the flux rope, which corresponds to the white solid contour in the left bottom panel. Various parameters can be readily obtained from these results. For example, this flux rope has a time range from 2007 November 7 (day 311 of 2007) to 2007 November 16 (day 320 of 2007), a scale size $\sim 0.4$ au, maximum axial field strength $\sim 0.28 \mathrm{nT}$, and right-hand chirality. This flux rope coincides with the structure $\mathrm{C}$ identified in the top panel.

The magnetic power spectral density (PSD) can be calculated by averaging the wavelet spectrum in the time domain. Figure 3 compares the PSD averaged over both upstream and downstream of the HTS. We selected a time interval from 2007 May 1 to 2007 August 30 to calculate the upstream PSD, and from 2007 August 30 to 2007 December 31 for the downstream PSD. We notice that such long intervals may eliminate some subtle structures in the PSD. This is because the continuous magnetic field is only available as daily 

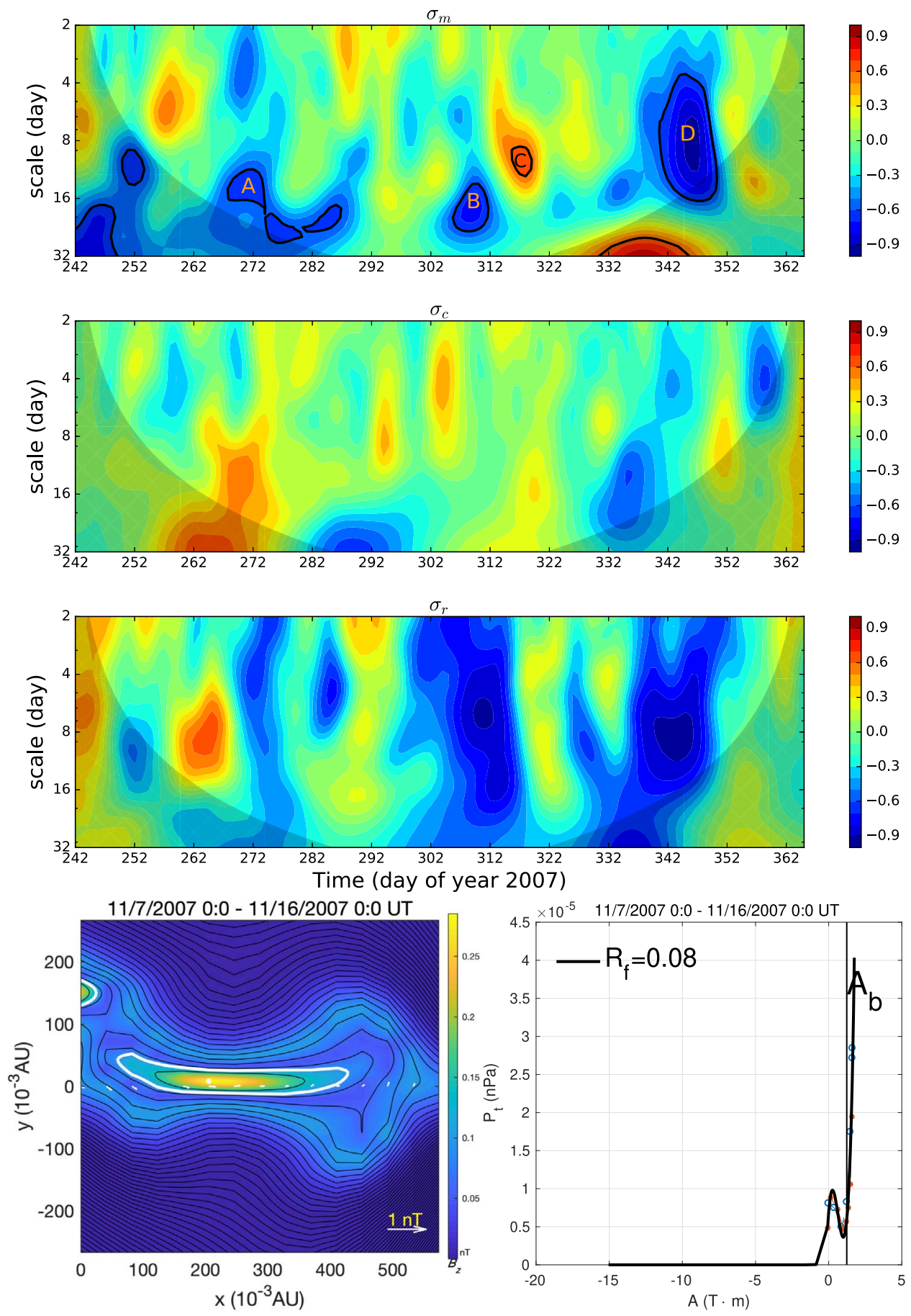

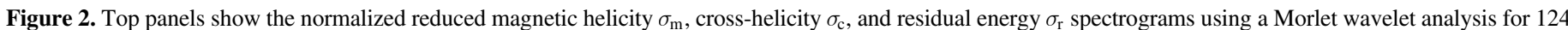

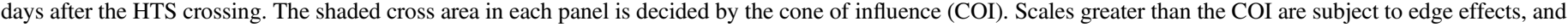
are not truly reliable. The bottom two panels show the reconstruction map and $P_{t}$ vs. $A$ curve of the GS reconstructed magnetic flux rope behind the HTS.

averaged data. Our upstream and downstream intervals both contain $\sim 120$ points. Further narrowing the intervals of wavelet analysis will make them contain too few points. Nevertheless, the fluctuation power is higher downstream than upstream, as the HTS amplifies the turbulence level and generates small-scale structures such as magnetic flux ropes. The upstream power spectrum does not show any apparent wave activity as is typical at quasi-parallel shocks (e.g., Zank et al. 2006a). Both upstream and downstream spectra exhibit a Kolmogorov-like $k^{-5 / 3}$ power-law shape. The magnetic power spectrum, along with the small cross-helicity and mostly small or negative residual energy downstream of the HTS, suggests that the downstream turbulence is dominated by $2 \mathrm{D}$ structures rather than Alfvén waves (Zank et al. 2015, 2017, 2018).

\section{Modeling the Observed ACR Fluxes}

Following Zhao et al. (2018), we apply the Zank et al. stochastic reconnection-based particle acceleration theory to fit the downstream ACR proton intensities measured by Voyager 2 in nine energy channels: $1.8-2.2,2.2-3.0,3.0-4.6,4.6-6.2$, 
Table 1

List of Possible Flux Ropes Downstream of the HTS

\begin{tabular}{lccccc}
\hline \hline No. & Central Time & Scale & Magnetic Helicity & \multicolumn{2}{c}{ Cross-helicity } \\
& (UT) & Residual Energy & $\sigma_{\mathrm{c}}$ & $\sigma_{\mathrm{r}}$ \\
\hline A & 2007 Oct 3 & 17 & -0.63 & 0.27 & -0.23 \\
B & 2007 Nov 5 & 18 & -0.69 & -0.04 & -0.66 \\
C & 2007 Nov 14 & 10 & 0.65 & -0.14 & -0.15 \\
D & 2007 Dec 12 & 8 & -0.77 & -0.78 \\
\hline
\end{tabular}

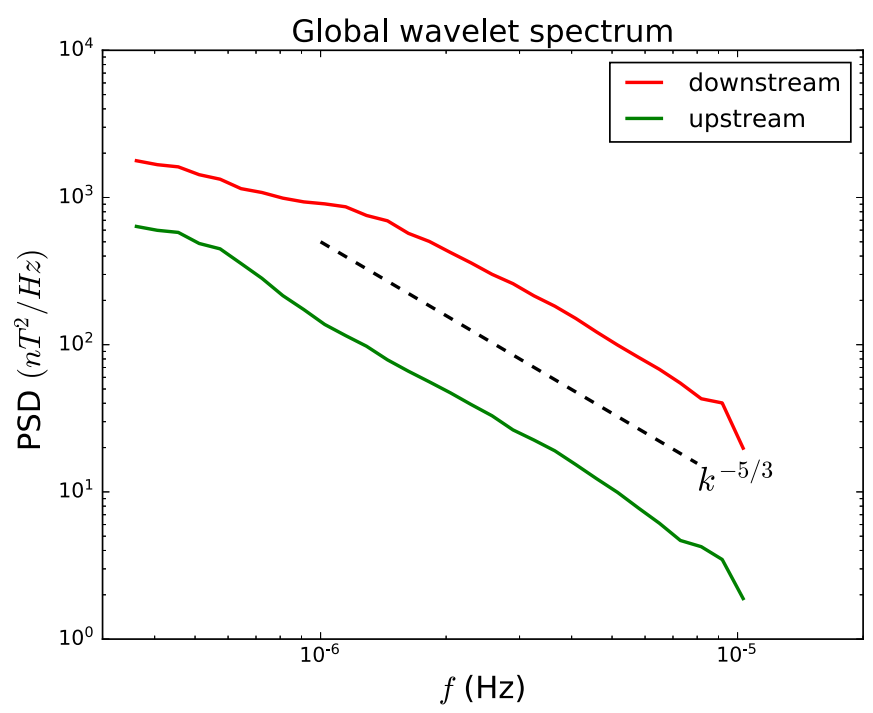

Figure 3. Wavelet magnetic power spectral density (PSD) averaged over upstream (green curve) and downstream (red curve). The dashed line displays a $k^{-5 / 3}$ spectrum as a reference.

6.2-7.7, 7.7-10.3, 10.3-12.8, 12.8-15.3, and 15.3-17.9 MeV. The corresponding average energies are 2.0, 2.6, 3.8, 5.4, $6.95,9.0,11.55,14.05$, and $16.6 \mathrm{MeV}$. Here, we consider two basic acceleration mechanisms for the downstream ACR proton energization: a first-order Fermi process due to magnetic island contraction, and a first-order Fermi process due to the reconnection electric field generated by island merging. The general solution for the particle differential intensity is given by Zank et al. (2014) and Zhao et al. (2018)

$$
\begin{gathered}
j\left(x, \frac{E}{E_{0}}\right)=j_{0}\left(\sqrt{\frac{E}{E_{0}}}\right)^{-\left(3+M_{E}+2 \tau_{d} /\left(3 \tau_{\mathrm{c}}\right) M_{E}^{2}\right) / 2} \\
\frac{E}{E_{0}} \exp \left[-\frac{\tau_{d}}{3 \tau_{\mathrm{c}}} M_{E} \frac{x}{L_{\mathrm{diff}}}\right] \\
I_{0}(\Phi) H\left(\ln \left(\sqrt{\frac{E}{E_{0}}}\right)\right) \\
H\left[\ln \sqrt{\frac{E}{E_{0}}}+\frac{1}{M_{E}} \frac{x}{L_{\mathrm{diff}}}\right] ; \\
\Phi=\sqrt{\frac{\tau_{d}}{3 \tau_{\mathrm{c}}} M_{E}^{2}\left(M_{E}-3+\frac{\tau_{d}}{3 \tau_{\mathrm{c}}} M_{E}^{2}\right)-\frac{\tau_{d}}{\tau_{e}} M_{E}} \\
{\left[\left(\ln \sqrt{\frac{E}{E_{0}}}\right)^{2}+\frac{2}{M_{E}} \ln \sqrt{\frac{E}{E_{0}}} \frac{x}{L_{\mathrm{diff}}}\right]^{1 / 2},}
\end{gathered}
$$

where $j_{0}$ is a normalization factor; $\tau_{\mathrm{c}}$ is the magnetic flux rope/ island contraction timescale; $\tau_{d}$ is the particle diffusion timescale; $\tau_{e}$ is the particle escape timescale; $L_{\mathrm{diff}}=U \tau_{d}$ is the diffusion length scale where $U$ is the solar wind speed; $M_{E}=U / V_{E}$ is a dimensionless parameter that characterizes the strength of the reconnection electric field where $V_{E}$ is a related characteristic velocity. $I_{0}$ denotes the zero-order modified Bessel function and $H$ is the Heaviside step function. The acceleration process depends on (i) the strength of the reconnection electric field $V_{E}$, (ii) the particle escape rate $\tau_{d} / \tau_{e}$, and (iii) the island contraction rate $\tau_{d} / \tau_{\mathrm{c}}$. The other three parameters $j_{0}, E_{0}$, and $L_{\mathrm{diff}}$ are used for normalization. Solution (7) is derived by assuming all these parameters are constant, and are energy independent.

The previous trial-and-error fitting was done simply without any estimates of the uncertainty (e.g., Zhao et al. 2018; Adhikari et al. 2019). Here, we employ a Monte Carlo Markov Chain (MCMC) technique to find a set of model parameters that best fit the data with uncertainties. The MCMC is a powerful technique for finding best-fit parameters and their associated confidence intervals in a model (e.g., Bonamente 2013). The basic idea is to search for parameters that maximize the likelihood of the model given some experimental data. At each step of the chain, a new set of parameters called candidates are drawn randomly from a proposal distribution. The candidates are accepted if the likelihood is larger than that of the previous step. When the likelihood is smaller than the previous step, the candidates are accepted according to the Metropolis-Hastings algorithm. After a sufficient number of iterations, the median values are taken as best-fit parameters. The confidence interval at a certain level can be approximated as the corresponding quantiles of the chain.

In principle, the MCMC technique requires the model parameters to be independent of one another. There are six parameters in our theoretical model, three of which are used for normalization and kept as fixed values in this study. These are the particle diffusion length scale $L_{\mathrm{diff}}=8.0 \times 10^{12} \mathrm{~cm}$, the particle injection energy $E_{0}=0.2 \mathrm{MeV}$, and the particle flux with injection energy $E_{0}$ at the injection point $j_{0}=7.0 \times$ $10^{3} \mathrm{~cm}^{-2} \mathrm{~s}^{-1} \mathrm{sr}^{-1} \mathrm{MeV}^{-1}$. The remaining three parameters $V_{E}, \tau_{d} / \tau_{\mathrm{c}}$, and $\tau_{d} / \tau_{e}$ are free parameters to be obtained from the MCMC technique. We choose a point $\sim 22$ days behind the shock as the injection point (on 2007 September 22) where low-energy particle fluxes are near a local minimum. For each set of parameters, we calculate the model predictions of the particle flux at all the times and energies that we consider. We assume that each measurement is distributed as a Gaussian random variable, so the likelihood of the model can be estimated by the chi-square: $\chi^{2}=\sum_{i}\left(y_{i}-\hat{y_{i}}\right)^{2} / \sigma_{i}^{2}$, where $y_{i}$ is the observed particle intensity, $\sigma_{i}$ is the uncertainty of the observation, and $\hat{y}_{i}$ is the model predicted flux.

Figure 4 shows the histograms of the three free model parameters $V_{E} / U, \tau_{d} / \tau_{\mathfrak{c}}$, and $\tau_{d} / \tau_{e}$ throughout the MCMC simulation. The chain was run for 10,000 steps, and the parameters exhibit approximately single-peaked distributions 
as suggested by the histograms. We find that the best-fit parameters with $68 \%$ confidence intervals are the following,

$$
\begin{aligned}
& V_{E} / U \in[0.055,0.058] ; \quad \tau_{d} / \tau_{\mathrm{c}} \in[0.161,0.173] ; \\
& \tau_{d} / \tau_{e} \in[6.165,6.267] .
\end{aligned}
$$

Here, we assume a constant solar wind velocity of $U=140 \mathrm{~km} \mathrm{~s}^{-1}$ in the inner heliosheath. The best-fit parameters are chosen as the median values,

$$
\begin{aligned}
V_{E} / U & =0.056 ; \quad \tau_{d} / \tau_{\mathrm{c}}=0.167 ; \\
\tau_{d} / \tau_{e} & =6.219 .
\end{aligned}
$$

The best-fit parameters suggest a relationship or ordering between the various timescales. For example, $\tau_{d}<\tau_{\mathrm{c}}$ suggests that the particle diffusion timescale is smaller than the island contraction timescale-this means that particles may be energized diffusively in the region containing multiple magnetic islands, because particles can diffuse between multiple islands during the time it takes for a flux rope to experience significant contraction. The relation $\tau_{e}<\tau_{d}$ suggests an efficient particle escape process compared to particle diffusion. This is common for the observed relatively soft particle spectrum for events in the inner heliosphere (Adhikari et al. 2019). The reduced chi-square that corresponds to the best-fit parameters is around 55. Ideally, the reduced chi-square should be about unity for a model that is consistent with the data. In our case, the reduced chi-square value is significantly large. This is because our model is not intended to explain finescale fluctuations in the energetic particle flux. In other words, there are intrinsic fluctuations that have not been taken into account by the model. It also should be pointed out that the values for the fixed parameters $L_{\mathrm{diff}}, E_{0}$, and $j_{0}$ are chosen quite arbitrarily. The parameter $L_{\text {diff }}$ may be justified by its definition $L_{\text {diff }}=\kappa / U$, where $\kappa$ is the diffusion coefficient for particles, and $U$ is the solar wind speed. Using an approximate solar wind speed in the heliosheath $U=140 \mathrm{~km} \mathrm{~s}^{-1}$, our choice of $L_{\text {diff }}$ translates to a diffusion coefficient of $\kappa=1.12 \times 10^{20} \mathrm{~cm}^{2} \mathrm{~s}^{-1}$. On the other hand, the diffusion coefficient is connected to the mean free path (mfp) by $\kappa=\lambda v / 3$, where $\lambda$ is the $\operatorname{mfp}$ and $v$ is the particle velocity. We use $3.66 \times 10^{7} \mathrm{~m} \mathrm{~s}^{-1}$ as a representative velocity in our considered energy range, which corresponds to $\sim 7 \mathrm{MeV}$, so that $\lambda=3 \kappa / v=0.9 \times 10^{9} \mathrm{~m} \sim 0.006$ au. We can also obtain other related dimensional parameters. The diffusion timescale is $\tau_{d}=L_{\text {diff }} / U=5.7 \times 10^{5} \mathrm{~s}$; the island contraction timescale is $\tau_{\mathrm{c}}=\tau_{d} /\left(\tau_{d} / \tau_{\mathrm{c}}\right)=3.4 \times 10^{6} \mathrm{~s}$; the escape timescale is $\tau_{e}=\tau_{d} /\left(\tau_{d} / \tau_{e}\right)=9.1 \times 10^{4} \mathrm{~s}$. The electric field parameter $M_{E}=\left(V_{E} / U\right)^{-1} \simeq 18$. The parameter $j_{0}$ is the intensity for particles with the injection energy $E_{0}$ at the injection point. Since we consider an injection energy $(0.2 \mathrm{MeV})$ far below the considered energy range, there are no data to justify the choice of the number directly. Nevertheless, the model does agree well with the observations qualitatively, as illustrated by Figure 5 where we overplot our model with best-fit parameters on the observed energetic particle flux.
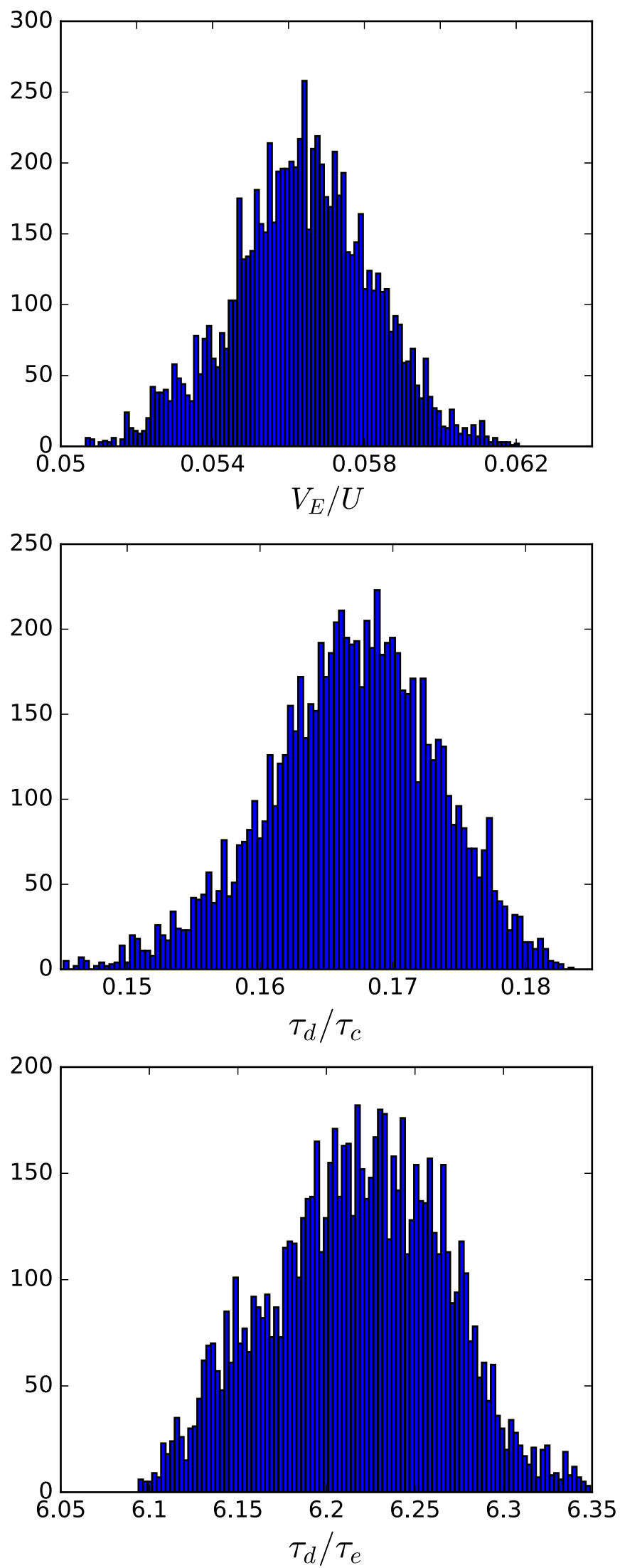

Figure 4. Histograms of $V_{E} / U, \tau_{d} / \tau_{\mathfrak{c}}$, and $\tau_{d} / \tau_{e}$ with 100 bins in the MCMC simulation. 


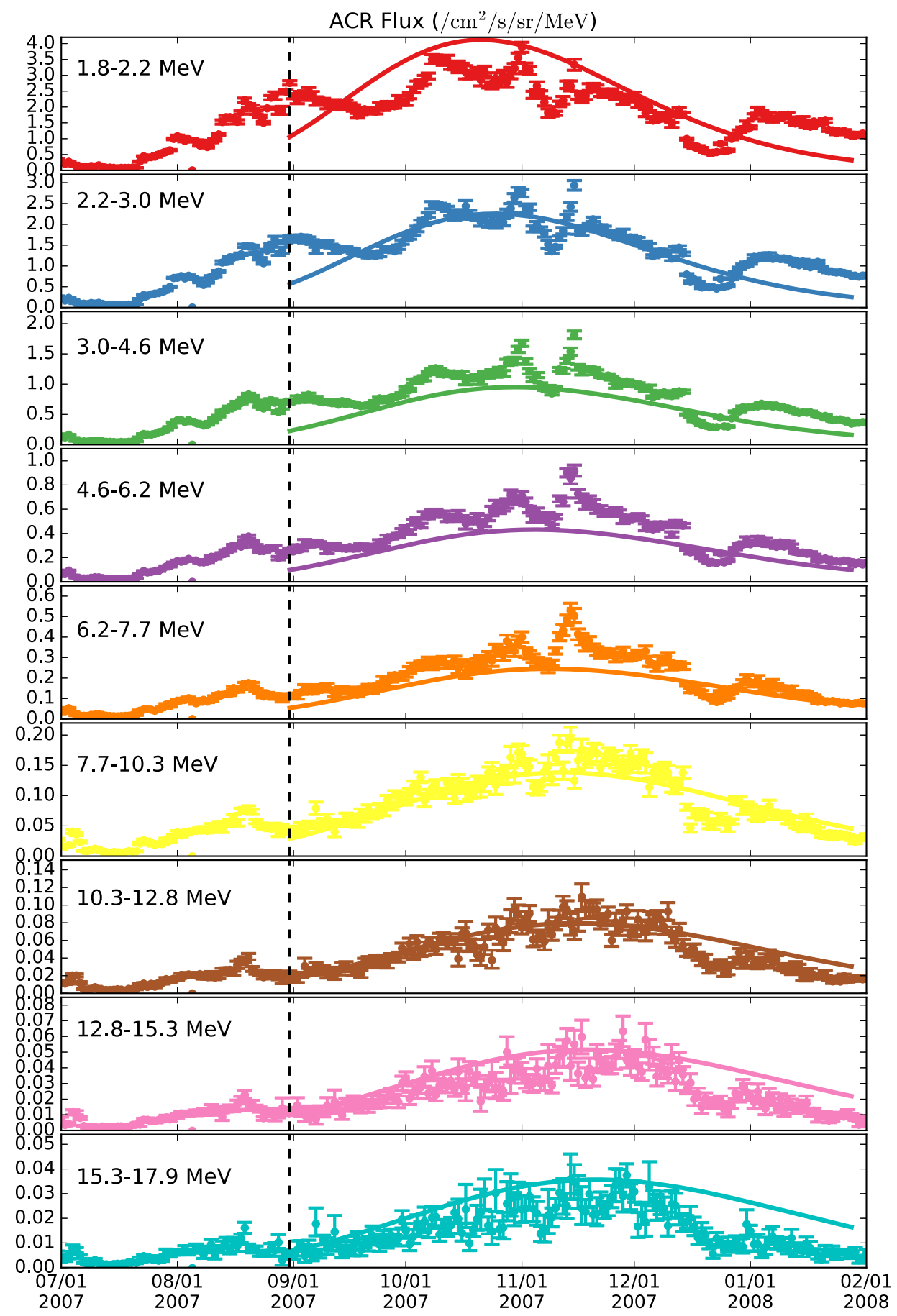

Figure 5. ACR proton flux evolution during the period from 2007 July 1 to 2008 February 1 . The uncertainties of the observed proton flux are plotted as error bars. The dashed vertical line represents the HTS crossing, and the smooth curves behind the HTS show our theoretical modeling results.

Figure 5 displays the time evolution of the ACR proton flux in the energy range $1.8-17.9 \mathrm{MeV}$ with a resolution of one day for the selected period from 2007 July 1 to 2008 February 1. The uncertainties of the observed proton flux are plotted as error bars, and are calculated from the observed counts $N$. For a large count (e.g., $N>20$ ), the uncertainty is calculated by $\frac{\sqrt{N}}{N} \times$ Flux. When the counts are smaller than 20 , the upper limit of the observed flux is estimated by $\frac{\sqrt{N+\frac{3}{4}}+1}{N} \times$ Flux, and the lower limit is $\frac{\sqrt{N-\frac{1}{4}}}{N} \times$ Flux (Gehrels 1986). The dashed vertical line identifies the HTS crossing by Voyager 2 . The curves of different colors behind the HTS are the predicted results of our model using the best fitting parameters for all nine energy bands. It is clear from the figure that our model does not fit well with the lower energy channels, but matches the higher energy bands. The downstream proton intensity of each energy channel is amplified relative to its value at the shock. For example, the largest amplification is about $\sim 2$ times for the $1.8-2.2 \mathrm{MeV}$ energy band and $\sim 4$ times for the 15.3-17.9 MeV energy channel. The flux amplification factor is in ascending order with increasing energy. The distance 

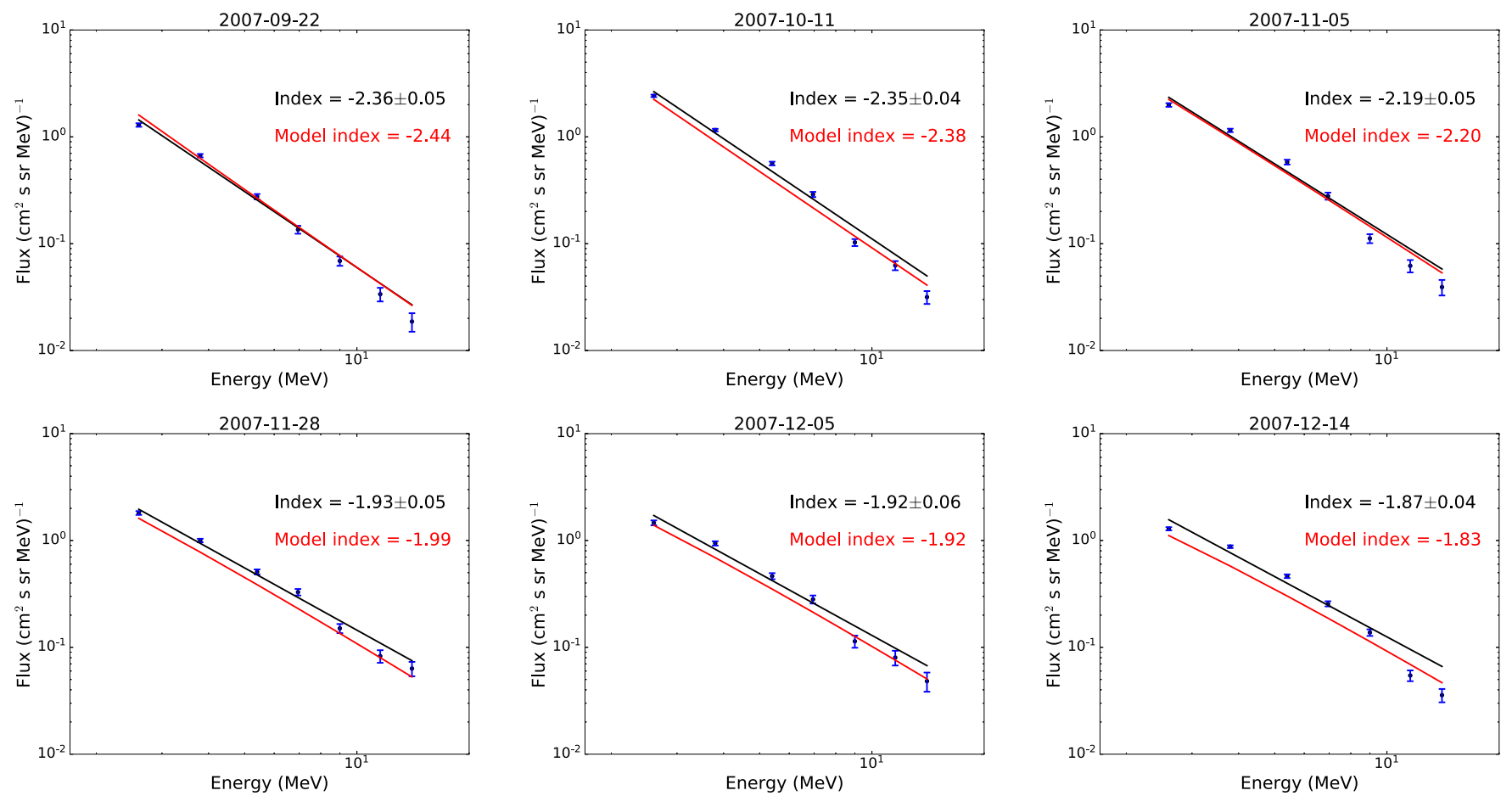

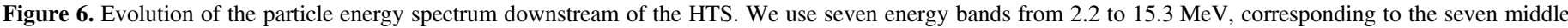

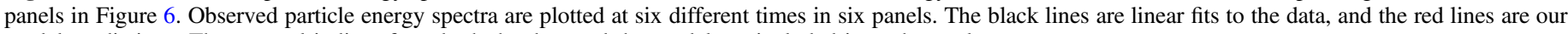
model predictions. The spectral indices from both the data and the model are included in each panel.

between particle flux peak and the HTS also increases with increasing particle energy, and high-energy particles $(>4.6$ $\mathrm{MeV}$ ) fluxes peak more than 2 months after the HTS crossing. These features are predicted by our reconnection-based particle acceleration model.

Figure 6 shows the evolution of the particle differential intensity spectra in the inner heliosheath region, which exhibits a power-law shape. The six representative instances in panels from top left to bottom right are organized in chronological order. Here, we choose seven energy bands from 2.2 to $15.3 \mathrm{MeV}$ and obtain the observed power-law index by applying a linear fit in $\log$ scale to the data as indicated by the black lines in each panel. Evidently, the particle spectrum becomes harder gradually as Voyager 2 crossed the HTS and continued to go deeper into the heliosheath. As shown by the red line in each panel, our theoretical model (7) produces a power-law-like distribution (Zank et al. 2014; Zhao et al. 2018), and an approximate power-law index can be obtained within the selected energy range. Our model predicted spectral indices agree well with the observational results. Both the theoretical model and observations show the hardening of the particle power-law spectra with increasing distance downstream of the HTS.

\section{Discussion and Conclusion}

We show Voyager 2 measurements of energetic proton flux, magnetic field, and plasma properties to investigate the acceleration of ACR protons in the inner heliosheath within 1 or 2 au of the HTS. All the data used in this paper have a resolution of one day. The enhancement of the ACR proton flux behind the HTS is inconsistent with the predictions of 1D steady-state DSA theory but can be explained by a reconnection-based stochastic particle acceleration mechanism associated with magnetic island dynamics in the heliosheath. This suggests that the source of the ACRs is not located at the HTS. The quasi-perpendicular HTS itself is responsible primarily for generating the downstream magnetic islands or flux ropes.

To find observational evidence of magnetic islands downstream of the HTS, we analyzed the spectra of the normalized reduced magnetic helicity, cross-helicity, and residual energy from the magnetic field and plasma fluctuations in the postHTS region. The location and scales of the structures are characterized using Morlet wavelet spectrograms of these turbulence quantities. Our results suggest the presence of magnetic flux ropes/islands, which is further confirmed by the GS reconstruction. Previous studies at 1-5 au have found numerous small-scale flux ropes (e.g., Zhao et al. 2018; Zheng $\& \mathrm{Hu} 2018$ ) in regions downstream of shocks. However, due to the limited resolution of the data in the heliosheath, the number of flux ropes that can be constructed from observation is not as large as might be predicted from numerical simulations (e.g., Drake et al. 2010). Nevertheless, our analysis provides evidence for the existence of possible flux rope structures in the post-HTS region.

Following Zhao et al. (2018), the enhancement of energetic proton flux downstream of the HTS is modeled by the Zank et al. kinetic transport theory for stochastic particle acceleration via reconnection processes associated with interacting magnetic islands (Zank et al. 2014). We improve the fitting procedure and apply the MCMC technique to find the best-fit parameters for the modeling. Our theoretical solution with the best-fit parameters agree reasonably well with the observed ACR proton flux and spectrum evolution in the region behind the HTS. The modeling results show that stochastic acceleration by interacting magnetic islands can explain the ACR proton peak beyond the HTS.

We emphasize that the magnetic reconnection mechanism explains the transient features of the accelerated charged 
particle spectrum within $\sim 1$ au downstream of the HTS, but not the large-scale variability of energetic particles between the HTS and the heliopause. Although the simplest 1D steady-state DSA model cannot reproduce all observational features, the possibility that downstream ACR fluxes might be more complicated than expected was already foreseen before the Voyager 1 crossing (Kóta \& Jokipii 2004) based on DSA at a 2D shock geometry. Our study does not rule out DSA as a possible source of ACRs and it is possible that the two mechanisms may work together, as suggested by Zank et al. (2015).

As Voyager 2 traveled deeper into the inner heliosheath, further from the HTS, Stone et al. (2017), Cummings et al. (2019) find that the ACR intensity is weakly anisotropic, being slightly stronger in the $-\mathrm{T}$ direction than the $\mathrm{N}$ direction. This can be understood in part on the basis of the blunt shock model (e.g., McComas \& Schwadron 2006; Kóta \& Jokipii 2008) because, as the spacecraft moves outward, it connects with magnetic field lines that are connected to regions of the HTS that are possibly further down the flanks toward the heliotail and perhaps at higher latitudes. Since these ACRs originate from different regions of the HTS, they stream away diffusively upstream and downstream from the shock. Consequently, because the diffusive streaming is along the magnetic field in the inner heliosheath, there should be a weak anisotropy in both the $-\mathrm{T}$ and $\mathrm{N}$ directions, which means that there exists a flow from the flank or tail of the heliosphere toward the nose and is slightly equatorward as well. However, the observed anisotropy does not determine whether it is DSA that is responsible for accelerating the particles elsewhere in the region of the HTS. The acceleration mechanism discussed here and in Zank et al. (2015) discusses the acceleration of ACRs in the region downstream of and relatively close to the HTS (within $\sim 1 \mathrm{au}$ ) due to the presence of magnetic islands generated either by the quasi-perpendicular shock or by reconnection in the downstream HTS. Since the HTS is perpendicular virtually everywhere on average, with the possible exception of the highest polar regions, and the wavy HCS circumscribes the ecliptic region of the HTS (likely extending to quite high latitudes), the acceleration of ACRs behind the HTS via magnetic flux ropes will occur across almost the entire HTS region from the nose to the flanks and heliotail regions. Consequently, because of the geometry of the magnetic field in the inner heliosheath, we would expect ACRs to stream diffusively away from these acceleration regions into the deeper heliosheath. If indeed particle acceleration is more efficient downstream of the HTS in the vicinity of the current sheet, then its possible that the anisotropy in the $-\mathrm{T}$ direction may be a little larger than in the $\mathrm{N}$ direction. This would need to be tested with a global heliospheric model however.

We note in closing that the presence of weak anisotropy does not contradict the reconnection mechanism for ACR acceleration. Indeed, because the observed ACR anisotropy is weak $(\sim 2 \%)$, the nearly isotropic assumption made in reducing the full transport equation to the spatial diffusion form used here (Zank et al. 2014) is valid. Since the theoretical model is only solved for the isotropic particle distribution, we cannot reproduce the observed ACR anisotropy. The work presented in this paper only explains why the ACR intensity did not peak right at the shock. We do not address the further unfolding and intensity growth in the ACR energy spectrum observed by the Voyagers as they moved to and $\sim 10$ au beyond the HTS. To summarize, we present observational evidence in the post-HTS region (within $\sim 1 \mathrm{au}$ ) that supports particle acceleration by magnetic reconnection-related processes associated with magnetic flux ropes.

We acknowledge the partial support of the NSF EPSCoR RII-Track-1 Cooperative Agreement OIA-1655280, and partial support from an NSF/DOE Partnership in Basic Plasma Science and Engineering via NSF grant PHY-1707247, a NASA IBEX grant Sub0000167/80NSSC18K0237, NSF grant AGS 1650854 and NASA HGI-80NSSC19K0276, and a NASA grant NNX15AI65G. A.C. and E.S. are supported by NASA grant NNN12AA01C. L.F.B. acknowledges partial support from NASA/GSFC Contract 80GSFC19C0012.

\section{ORCID iDs}

L.-L. Zhao (1) https://orcid.org/0000-0002-4299-0490

G. P. Zank (iD https://orcid.org/0000-0002-4642-6192

Q. Hu (iD https://orcid.org/0000-0002-7570-2301

Y. Chen (iD https://orcid.org/0000-0002-0065-7622

L. Adhikari (i) https://orcid.org/0000-0003-1549-5256

J. A. leRoux (iD https://orcid.org/0000-0001-9199-2890

A. Cummings (D) https://orcid.org/0000-0002-3840-7696

L. F. Burlaga (D) https://orcid.org/0000-0002-5569-1553

\section{References}

Adhikari, L., Khabarova, O., Zank, G. P., et al. 2019, ApJ, 873, 72 Adhikari, L., Zank, G. P., Hunana, P., et al. 2017, ApJ, 841, 85

Bonamente, M. 2013, Statistics and Analysis of Scientific Data (New York: Springer)

Burlaga, L. F., \& Ness, N. F. 2009, ApJ, 703, 311

Burlaga, L. F., Ness, N. F., Acuña, M. H., et al. 2005, Sci, 309, 2027

Burlaga, L. F., Ness, N. F., \& Acuña, M. H. 2006, ApJ, 642, 584

Burlaga, L. F., Ness, N. F., Acuña, M. H., et al. 2008, Natur, 454, 75

Burlaga, L. F., Ness, N. F., Acuña, M. H., et al. 2009a, ApJ, 692, 1125

Burlaga, L. F., Ness, N. F., \& Acuña, M. H. 2009b, ApJL, 691, L82

Chen, Y., Hu, Q., \& le Roux, J. A. 2019, ApJ, 881, 58

Cummings, A., \& Stone, E. 2008, Proc. ICRC, 1, 827

Cummings, A., \& Stone, E. 2013, in AIP Conf. Proc. 1516, Centenary Symposium 2012: Discovery of Cosmic Rays, ed. J. F. Ormes (Melville, NY: AIP), 97

Cummings, A., Stone, E., Heikkila, B. C., et al. 2019, Proc. ICRC, 36, 1071

Decker, R. B., Krimigis, S. M., Roelof, E. C., et al. 2005, Sci, 309, 2020

Drake, J. F., Opher, M., Swisdak, M., \& Chamoun, J. 2010, ApJ, 709, 963

Ferreira, S. E. S., Potgieter, M. S., \& Scherer, K. 2007, JGR, 112, A11101

Fisk, L. A., \& Gloeckler, G. 2009, AdSpR, 43, 1471

Florinski, V., Decker, R. B., le Roux, J. A., et al. 2009, GeoRL, 36, L12101

Gehrels, N. 1986, ApJ, 303, 336

Hill, M. E., Decker, R. B., Brown, L. E., et al. 2014, ApJ, 781, 94

Hu, Q., Zheng, J., Chen, Y., le Roux, J., \& Zhao, L. 2018, ApJS, 239, 12

Khabarova, O., Zank, G. P., Li, G., et al. 2015, ApJ, 808, 181

Khabarova, O. V., \& Zank, G. P. 2017, ApJ, 843, 4

Kóta, J., \& Jokipii, J. R. 2004, AIP Conf. Proc. 719, Physics of the Outer Heliosphere (Melville, NY: AIP), 272

Kóta, J., \& Jokipii, J. R. 2008, in AIP Conf. Proc. 1039, Particle Acceleration and Transport in the Heliosphere and Beyond, ed. G. Li et al. (Melville, NY: AIP), 397

le Roux, J. A., Zank, G. P., \& Khabarova, O. V. 2018, ApJ, 864, 158

le Roux, J. A., Zank, G. P., Webb, G. M., \& Khabarova, O. 2015, ApJ, 801,112

le Roux, J. A., Zank, G. P., Webb, G. M., \& Khabarova, O. V. 2016, ApJ, 827,47

Matthaeus, W. H., Goldstein, M. L., \& Smith, C. 1982, PhRvL, 48, 1256

McComas, D. J., \& Schwadron, N. A. 2006, GeoRL, 33, L04102

Moldwin, M. B., Phillips, J. L., Gosling, J. T., et al. 1995, JGR, 100, 19903

Mostafavi, P., Zank, G. P., \& Webb, G. M. 2017, ApJ, 841, 4

Mostafavi, P., Zank, G. P., \& Webb, G. M. 2018, ApJ, 868, 120

Pesses, M. E., Eichler, D., \& Jokipii, J. R. 1981, ApJL, 246, L85

Senanayake, U. K., Florinski, V., Cummings, A. C., et al. 2015, ApJ, 804, 12 
Stone, E., Cummings, A. C., Heikkila, B. C., et al. 2017, Proc. ICRC, 301, 57

Stone, E. C., Cummings, A. C., McDonald, F. B., et al. 2005, Sci, 309, 2017

Stone, E. C., Cummings, A. C., McDonald, F. B., et al. 2008, Natur, 454, 71

Telloni, D., Bruno, R., D’Amicis, R., et al. 2012, ApJ, 751, 19

Telloni, D., Perri, S., Bruno, R., et al. 2013, ApJ, 776, 3

Torrence, C., \& Compo, G. P. 1998, BAMS, 79, 61

Vasquez, B. J., Markovskii, S. A., \& Smith, C. W. 2018, ApJ, 855, 121

Zank, G. P., Adhikari, L., Hunana, P., et al. 2017, ApJ, 835, 147

Zank, G. P., Adhikari, L., Zhao, L.-L., et al. 2018, ApJ, 869, 23

Zank, G. P., Dosch, A., Hunana, P., et al. 2012, ApJ, 745, 35

Zank, G. P., Heerikhuisen, J., Pogorelov, N. V., Burrows, R., \& McComas, D. 2010, ApJ, 708, 1092

Zank, G. P., Hunana, P., Mostafavi, P., et al. 2015, ApJ, 814, 137
Zank, G. P., le Roux, J. A., Webb, G. M., Dosch, A., \& Khabarova, O. 2014, ApJ, 797, 28

Zank, G. P., Li, G., Florinski, V., et al. 2006a, JGRA, 111, A6

Zank, G. P., Pauls, H. L., Williams, L. L., et al. 1996, JGR, 101, 21639

Zank, G. P., Shaikh, D., \& Ao, X. 2006b, in AIP Conf. Ser. 858, Physics of the Inner Heliosheath: Voyager Observations, Theory, and Future Prospects, ed. J. Heerikhuisen (Melville, NY: AIP), 308

Zhang, M. 2006, in AIP Conf. Proc. 858, Physics of the Inner Heliosheath: Voyager Observations, Theory, and Future Prospects, ed. J. Heerikhuisen (Melville, NY: AIP), 226

Zhao, L.-L., Zank, G. P., \& Adhikari, L. 2019a, ApJ, 879, 32

Zhao, L.-L., Zank, G. P., Chen, Y., et al. 2019b, ApJ, 872, 4

Zhao, L. L., Zank, G. P., Khabarova, O., et al. 2018, ApJL, 864, L34

Zheng, J., \& Hu, Q. 2018, ApJL, 852, L23 